\title{
Assessment of drug related problems among type 2 diabetes mellitus patients with hypertension in Hiwot Fana Specialized University Hospital, Harar, Eastern Ethiopia
}

\author{
Yohanes Ayele $2^{2^{*}} \mathbb{0}$, Kibkab Melaku, Mesay Dechasa², Mohammed Biset Ayalew ${ }^{3}$ and Boressa Adugna Horsa ${ }^{3}$
}

\begin{abstract}
Objective: This study was conducted to assess magnitude and pattern of drug related problems among patients with type 2 diabetes mellitus (T2DM) and hypertension.

Results: This study identified 364 drug related problems (DRPs) across the three categories of drug related problems, giving an average of 1.8 DRPs per patient. The effect of drug treatment being not optimal 179 (49.2\%), untreated indication and symptoms 77 (21.1\%), unnecessary drug-treatment 39 (10.7\%) and adverse drug reactions 69 (19\%) were the most frequent categories of DRPs identified. In general, high prevalence of drug-related problems was identified among patients with T2DM hypertension. The effect of drug treatment being not optimal, untreated indication and symptoms, unnecessary drug-treatment and adverse drug reactions were the most frequent categories of drug related problems identified. Therefore, the clinicians should work to improve patient care through prevention and resolving drug related problems since it can affect the quality of the care significantly.
\end{abstract}

Keywords: Drug related problems, Type 2 diabetic mellitus, Hypertension

\section{Introduction}

Diabetes mellitus is a group of metabolic disorders caused either by the inability to produce insulin or by the body not being able to use insulin effectively or both [1]. Globally, 415 million adults are estimated to have diabetes currently and there are 318 million adults with impaired glucose tolerance which puts them at high risk of developing the disease in the future. Developing countries is suffering from increasing burden of diabetes mellitus $[1,2]$. In 2015 , it was estimated that 9.5-29.3 million peoples live with diabetes in the Africa Region [3].

American diabetic association classification categorizes diabetes into four categories; type 1, type 2, gestational and other diabetes [4]. T2DM accounts for $90-95 \%$ of the incidence of diabetes and is associated with a strong

\footnotetext{
*Correspondence: yohanes.ayele16@gmail.com

2 Department of Clinical Pharmacy, School of Pharmacy, College of Health and Medical Sciences, Haramaya University, P.O.Box 235, Harar, Ethiopia Full list of author information is available at the end of the article
}

genetic predisposition as well as age, obesity and lack of physical activity [5]. Hypertension is a common comorbidity in T2DM patients with a prevalence of up to twothirds of the population and it may be present by the time T2DM is diagnosed or even before the onset of hyperglycaemia [6]. The coexistence of hypertension and T2DM often indicates more severe disease and increases risk of macro vascular and microvascular complications [7-9].

Effective diabetes management often presents enormous challenges. Not surprisingly, clinicians and patients can be overwhelmed by the need to address comorbid chronic conditions in addition to patients' diabetes-specific treatment goals. Furthermore, during management undesirable event which involves or is suspected to relate to drug therapy may occur, which is often termed as DRPs $[10,11]$.

DRPs could prevent or delay patients from achieving desired therapeutic goals and it has been attributed to unnecessary over prescription of drugs, substantial 
worsening of diseases, avoidable increases in hospital admission rates, and longer hospital stays leading to a significant medical burden [12, 13]. However, DRPs are preventable in most cases [14]. Hence understanding the magnitude of the DRPs and pattern of its occurrence would be important input for the practitioner in the process of DM management particularly in the patients with coexisting disease. Therefore, this study was conducted to assess the prevalence and pattern of DRPs among patients with T2DM and Hypertension in Hiwot Fana Specialized University Hospital (HFSUH), Eastern Ethiopia.

\section{Main text \\ Methods}

\section{Study design and settings}

Institution based retrospective cross-sectional study was conducted in HFSUH in Harar city from May to 30 August, 2017.

\section{Study population}

All diabetic patients on chronic follow up at HFSUH were the source populations. All adult ( $>18$ years) patients with T2DM and hypertension and on follow up during data collection period were study populations. Patients with missing or incomplete data were excluded.

\section{Sample size determination and sampling technique}

The actual sample size for the study was determined using the formula for single population proportion. From HFSUH data the number of diabetic patient uses as follow up in HFSUH was 356, the minimum sample size required for study was determined to 203 individuals.

\section{Data collection, processing and analysis}

The record of patients with types 2 diabetes and hypertension and currently on follow up was identified until the targeted sample size was achieved. The data was collected using data extraction format. The format was designed to collect information on sociodemographic characteristics of patients, disease characteristics of patients and medications. The collected data was revised for its completeness and consistency before it was entered into epi-data and processed and analyzed using SPSS version 20 . The findings were presented using summary measures and displayed using tables.

\section{Evaluation of drug related problems and classification}

There have been different approaches to classify and summarize DRPs. However, few of these systems are validated and reliable [15]. We used the Pharmaceutical Care Network Europe Classification scheme for drugrelated problems V8.02 [13]. Identification of DRPs was assessed by two clinical pharmacists who were trained on study protocol developed based on the Drug Information Handbook [16], standards of medical care in diabetes 2017 [17] and Joint National Committee (JNC) 8 [18].

\section{Operational definition}

Nephropathy: Presence of albuminuria which defined as urine albumin to-creatinine ratio $\geq 30 \mathrm{mg} / \mathrm{g}$ creatinine measured in two of three specimens collected within a 3to 6-month period [4].

Retinopathy: Presence of clinically significant macular edema, severe non-proliferative or proliferative retinopathy seen under high-quality fundus photographs [4].

Neuropathy: Diverse clinical manifestations including diabetic peripheral neuropathy, autonomic neuropathy, cardiovascular autonomic neuropathy, and gastrointestinal neuropathies [4].

\section{Results}

Socio-demographic characteristics of the Patients

A total of 203 patients were included in this study out of which 107 (52.7\%) were female and 96 (47.3\%) were male. The majority 137 (67.5) patients' lives in urban area and $139(68.5 \%)$ patients are married (Table 1$)$.

\section{Disease characteristics of patients}

More than half 109 (53.7\%) of patients' lived with diabetes for 6-10 years, 62 (30.5\%) lived with diabetes for less

Table 1 The demographic characteristic of patients with T2DM and HTN in Hiwot Fana Specialized University Hospital, $2017(n=203)$

\begin{tabular}{lr}
\hline Variables & $\mathbf{n}(\%)$ \\
\hline Sex & \\
Male & $96(47.3)$ \\
Female & $107(52.7)$ \\
Age & \\
$>50$ & $158(77.8)$ \\
$\leq 50$ & $45(22.2)$ \\
Residents & \\
Urban & $137(67.5)$ \\
Rural & $66(32.5)$ \\
Marital status & \\
Married & $139(68.5)$ \\
Single & $64(31.5)$ \\
Smoking & \\
Non-smoker & $113(55.7)$ \\
Smoker & $90(44.3)$ \\
Alcohol consumption & \\
Yes & $63(31.0)$ \\
No & $140(69.0)$ \\
\hline
\end{tabular}


than 5 years. The mean T2DM duration was 7.4 years. Regarding to fasting blood sugar, a large portion (43.3\%) of the patients 88 had blood glucose level of greater than $180 \mathrm{mg} / \mathrm{dL}$ in their last visit. Concerning to HTN, the $B P$ level at last visit were less than or equal to $140 / 80$ for 138 (67.9\%) patients whereas $65(32.1 \%)$ patient had BP above $140 / 80 \mathrm{mmHg}$. In this study, $122(60.1 \%)$ patients have comorbidity other than HTN. Moreover, about 71\% of patients developed T2DM related complications. The most prevalent T2DM related complication were Neuropathy 45 (22.2\%) followed by Retinopathy 25 (12.3\%) (Table 2).

\section{Medications used in patients with type 2 diabetes mellitus and hypertension}

In this study, 93 (45.8\%) patients received monotherapy out of which 70 (34.5\%) were metformin, 16 (7.9\%) insulin and 7 (3.4\%) glibenclamide. More than half of the patients received dual therapy; metformin and glibenclamide 21 (19\%), insulin and metformin 21 (10.3\%) and insulin and glibenclamide 11 (5.4\%). Regarding HTN

Table 2 The disease characteristic of patients with T2DM and HTN in Hiwot Fana Specialized University Hospital, $2017(n=203)$

\begin{tabular}{lr}
\hline Disease characteristics & $\mathbf{n}(\%)$ \\
\hline Duration of DM (years) & $62(30.5)$ \\
$<5$ & $109(53.7)$ \\
$\geq 5-10$ & $32(15.8)$ \\
$\geq 10$ & \\
FBS at last visit (mg/dL) & $88(43.3)$ \\
$>180$ & $45(22.2)$ \\
140-180 & $68(33.5)$ \\
$<140$ & \\
BP at last visit & $138(67.9)$ \\
$\leq 140 / 80$ & $65(32.1)$ \\
$>140 / 80$ & \\
Comorbidity other than HTN ( $\mathrm{n}=122)$ & $47(38.6)$ \\
Ischemic heart disease (IHD) & $31(25.4)$ \\
Dyslipidaemia & $18(14.7)$ \\
Heart failure & $14(11.5)$ \\
Peripheral arterial disease & $12(9.8)$ \\
Other* & \\
T2DM complications ( $\mathrm{n}=144)$ & $45(31.2)$ \\
Neuropathy & $31(21.5)$ \\
Neuropathy and retinopathy & $25(17.4)$ \\
Retinopathy & $23(16.0)$ \\
Nephropathy & $20(13.9)$ \\
Diabetic foot ulcer &
\end{tabular}

FBS fasting blood sugar, $B P$ blood pressure

a Urinary tract infection, chronic obstructive pulmonary disease, acute pyelonephritis, portal HTN management, $160(78.8 \%)$ patients received medication for hypertension. A large share of patients 93 (58.1\%) received mono therapy. The angiotensin converting enzyme inhibitors (ACEIs) particularly enalapril were the most frequently used drug followed by the calcium channel blockers (CCBs) (Table 3).

\section{Drug related problems in type 2 diabetic with hypertension patients}

In this study, 364 DRPs were identified which is about 1.8 DRPs per patient. Among this, 179 (49.2\%) patients were not on optimal drug treatment. It was found that 25 stage II hypertensive patients received monotherapy whereas 133 patients did not achieve their glycaemic goal. Seventy-seven patients did not get drug therapy for a clear indications and symptoms. Forty-two T2DM complications were not treated and 29 hypertensive patients did not receive the antihypertensive therapy. In 39 (10.7\%) cases, there was unnecessary therapy. Fourteen stage I hypertensive patients received dual therapy while the appropriate therapy should have been a mono therapy. Sixty-nine cases of adverse reactions were reported and antidiabetic drugs were associated with 43 of all the cases.

\section{Discussion}

In current study, 364 DRPs were recorded which is about 1.8 DRPs per patient. This finding is lower than study conducted among Jordan [19] ambulatory patients where 11.2 DRPs per patient were reported but higher than study conducted in Nigeria [20] and southern Ethiopia [21]. The large variation seen on magnitude of DRPs across studies might be due to different classification of system used to classify DRPs and settings in which DRPs were assessed. Despite the difference seen in magnitude of DRPs, the finding in our study shows the need to make intervention on the DRPs so that the patients improve success of treatment outcomes. Furthermore we recommend future studies to use similar DRPs classification system to generate comparable evidences.

For patients with diabetes and HTN a target reduction of BP of $\leq 130 / 80 \mathrm{mmHg}$ is recommended [4]. Achieving this target is often challenging and patients need one or more drugs to achieve this goal. The poor BP control reported in this study might be explained by the patients not receiving optimal therapy. This result is in agreement with study conducted in Jordan [19] and Southern Ethiopia [21] where patient being not in optimal therapy was most commonly observed DRP.

Similarly, the American diabetic association (ADA) guideline recommends Pre-prandial plasma glucose target goal of $180-130 \mathrm{mg} / \mathrm{dL}$ is although the stringency varies based on the patient factors. In this study, 133 T2DM 


\begin{tabular}{lc} 
Table 3 The characteristics of medications used \\
to manage patients with T2DM and HTN in Hiwot Fana \\
Specialized University Hospital, 2017 (n= 203) \\
\hline Medication characteristics & $\mathbf{n}(\%)$ \\
\hline DM medication & \\
Metformin + glibenclamide & $78(38.4)$ \\
Metformin & $70(34.5)$ \\
Insulin + metformin & $21(10.3)$ \\
Insulin & $16(7.9)$ \\
Insulin + glibenclamide & $11(5.4)$ \\
Glibenclamide & $7(3.4)$ \\
Classes of HTN medication used & \\
ACEls & $41(20.2)$ \\
CCBs & $29(14.3)$ \\
Diuretics & $15(7.4)$ \\
Beta blockers & $4(2.0)$ \\
Centrally acting a $a_{2}$ agonist & $4(2.0)$ \\
Combined classes & $67(33.0)$ \\
ADR reported & \\
Gastrointestinal upset & $36(17.7)$ \\
Dry cough & $24(11.8)$ \\
Hypoglycaemia & $7(3.4)$ \\
Ankle oedema & $2(1.0)$ \\
\hline ACEls angoten
\end{tabular}

ACEls angiotensin converting enzyme inhibitors, CCBs calcium channel blockers

patients did not achieve the desired glycaemic goal. Although poor glycaemic control could be attributed to different factors such as life style modification, presence of other comorbidities; DRPs particularly non-adherence could be a major factor in this population. This in turn could be related to forgetfulness, refusal to take medication, cost of medication and unavailability $[22,23]$. Hence the health care providers should consider the issue of adherence to medication as important component T2DM patients' management.

The second most frequently observed DRP in present study is untreated symptoms and indication. Evidence indicates that patients with T2DM and HTN require aggressive medication therapy in order to halt and prevent morbidity, mortality and unnecessary hospital stay and disability $[4,18]$. However, in this study 28 stage I hypertensive patients' and one stage II hypertensive patient did not receives antihypertensive therapy. Moreover, there were also other comorbidities and DM related complications which were not given drug therapy.

In agreement with study conducted in other settings $[24,25]$, use of unnecessary drugs is commonly observed DRPs. In this study, 39 patients received unnecessary medication therapy for a clinical condition in which a single therapy suffice or for the condition that is not existed. Some drugs were prescribed without any clear indication.
These add on medications should be used only when there is clear indication because of risk of increased pill burden which in turn could lead to other DRPs.

Adverse drug reactions were also observed in this study. Hypoglycaemia is commonly observed adverse effect among patients receiving oral anti-diabetic drugs or insulin [26]. In this study, a few case of hypoglycaemia is observed and patients should be educated about the sign and symptoms of hypoglycaemia and its management. Antihypertensive agents particularly adverse reactions related to ACEIs were frequently reported and this finding was clearly demonstrated in this study [27]. In such situation angiotensin receptor blockers (ARBs) are recommended however there were no such drugs found in the patients record and this could be attributed to the absence of the drugs in the local market and its cost.

\section{Conclusion}

In the present study, high prevalence of drug-related problems was identified among patients with T2DM and hypertension. The effect of drug treatment being not optimal, untreated indication and symptoms, unnecessary drug-treatment and adverse drug reactions were the most frequent categories of DRPs identified. Therefore, the clinicians should work to improve patient care through prevention and resolving drug related problems since it can affect the quality of the care significantly.

\section{Limitation}

This study used retrospective study design hence it was relying only on information available in the patient records. Laboratory tests used in this study might not be the best methods to monitor blood glucose level. Organ function tests should have been used to determine renal clearance level and hepatic function. In addition, there might be unrecorded ADRs. Therefore, our assessment may not have been comprehensive enough to generate adequate information should generalized cautiously.

\section{Abbreviations \\ HFSUH: Hiwot Fana Specialized University Hospital; T2DM: types 2 diabetes mellitus; HNT: hypertension; DRP: drug related problem; ADR: adverse drug reaction; ACEls: angiotensin converting enzyme inhibitors; CCBs: calcium channel blockers; ADA: American diabetic association; ARBs: angiotensin receptor blockers.}

\section{Authors' contributions}

$\mathrm{KM}$ is principal author responsible for designing the study, data collection and analysis and interpretation of findings. YA is responsible for conception of the research idea and interpretation of findings and authored the manuscript. MD, MBA and BAB participated in study design, data collection, analysis and interpretation of the findings. All authors read and approved the final manuscript.

\footnotetext{
Author details

${ }^{1}$ Hiwot Fana Specialized University Hospital, Harar, Ethiopia. ${ }^{2}$ Department of Clinical Pharmacy, School of Pharmacy, College of Health and Medical Sciences, Haramaya University, P.O.Box 235, Harar, Ethiopia. ${ }^{3}$ Department
} 
of Clinical Pharmacy, School of Pharmacy, College of Medicine and Health Sciences, University of Gondar, Gondar, Ethiopia.

\section{Acknowledgements}

We would like to thank Hiwot Fana Specialized University Hospital staffs who supported us during data collection. In addition, we appreciate the data collectors who showed the utmost effort in acquiring appropriate information.

\section{Competing interests}

The author declare that they have no competing interests.

\section{Availability of data and materials}

The datasets are available from the corresponding author on reasonable request.

\section{Consent to publish}

Not applicable since there is no individual details in our result.

\section{Ethics approval and consent to participate}

Letter of ethical clearance was obtained from Haramaya University, college of medical and health science and submitted to the HFSUH to obtain permission to conduct the research. The confidentiality of the data collected was maintained throughout data processing.

\section{Funding}

The authors did not receive any fund for this research.

\section{Publisher's Note}

Springer Nature remains neutral with regard to jurisdictional claims in published maps and institutional affiliations.

Received: 23 September 2018 Accepted: 9 October 2018

Published online: 12 October 2018

\section{References}

1. World Health Organization. Global report on diabetes. Geneva: World Health Organization; 2017.

2. Animaw W, Seyoum Y. Increasing prevalence of diabetes mellitus in a developing country and its related factors. PLOS ONE. 2017:12(11):e0187670.

3. International Diabetic Federation. IDF diabetes atlas. 7th ed. Brussels: International Diabetic Federation; 2015.

4. American Diabetic Association. Standards of medical care in diabetes-2014. Diabetes Care. 2014:37(Supplement 1):S14-80.

5. Zheng Y, Ley SH, Hu FB. Global aetiology and epidemiology of type 2 diabetes mellitus and its complications. Nat Rev Endocrinol. 2018;14(2):88-98.

6. Colosia AD, Palencia R, Khan S. Prevalence of hypertension and obesity in patients with type 2 diabetes mellitus in observational studies: a systematic literature review. Diabetes Metab Syndr Obes Targets Ther 2013;6:327-38.

7. Hurst C, Thinkhamrop B, Tran HT. The association between hypertension comorbidity and microvascular complications in type 2 diabetes patients: a nationwide cross-sectional study in Thailand. Diabetes Metab J. 2015:39(5):395-404

8. Lastra G, Syed S, Kurukulasuriya LR, Manrique C, Sowers JR. Type 2 diabetes mellitus and hypertension: an update. Endocrinol Metab Clin North Am. 2014;43(1):103-22.
9. Bretzel RG. Comorbidity of diabetes mellitus and hypertension in the clinical setting: a review of prevalence, pathophysiology, and treatment perspectives. Clin Ther. 2007;29:S35-43.

10. Piette JD, Kerr EA. The impact of comorbid chronic conditions on diabetes care. Diabetes Care. 2006:29:725-31.

11. Pharmaceutical Care Network Europe. PCNE Classification scheme for Drug-Related Problems V8.02. [cited may 2017]. https://www.pcne.org/ working-groups/2/drug-related-problemclassification.

12. Fernandez-Llimos F, Faus MJ. From "drug-related problems" to "negative clinical outcomes". Am J Health Syst Pharm. 2005;62(22):2348-50.

13. Gastelurrutia P, Benrimoj SI, Espejo J, Tuneu L, Mangues MA, Bayes-Genis A. Negative clinical outcomes associated with drug-related problems in heart failure (HF) outpatients: impact of a pharmacist in a multidisciplinary HF clinic. J Card Fail. 2011;17(3):217-23.

14. Kaufmann CP, Stämpfli D, Hersberger KE, Lampert ML. Determination of risk factors for drug-related problems: a multidisciplinary triangulation process. BMJ Open. 2015;5(3):e006376

15. van Mil JW, Westerlund LO, Hersberger KE, Schaefer MA. Drug-related problem classification systems. Ann Pharmacother. 2004;38(5):859-67.

16. Lacy CF, Armstrong LL, Goldman MP, Snippet LLL. Drug information handbook. 25th ed. Hudson: Lexicomp; 2016.

17. American Diabetes A. Standards of medical care in diabetes-2017 abridged for primary care providers. Clin Diabetes. 2017;35(1):5-26.

18. James PA, Oparil S, Carter BL, Cushman WC, Dennison-Himmelfarb C, Handler J, et al. 2014 evidence-based guideline for the management of high blood pressure in adults: report from the panel members appointed to the Eighth Joint National Committee (JNC 8). JAMA 2014;311(5):507-20.

19. Al-Azzam SI, Alzoubi KH, AbuRuz S, Alefan Q. Drug-related problems in a sample of outpatients with chronic diseases: a cross-sectional study from Jordan. Ther Clin Risk Manag. 2016:12:233-9.

20. Ogbonna B, Amagba C. Investigation of drug therapy problems in type 2 diabetes outpatients with comorbid hypertension in a tertiary hospital in southeast Nigeria. Value Health. 2017;20(9):A859.

21. Koyra HC, Tuka SB, Tufa EG. Epidemiology and predictors of drug therapy problems among type 2 diabetic patients at Wolaita Soddo University teaching hospital, southern Ethiopia. Am J Pharmacol Sci. 2017;5(2):40-8.

22. Cramer JA, Benedict A, Muszbek N, Keskinaslan A, Khan Z. The significance of compliance and persistence in the treatment of diabetes, hypertension and dyslipidaemia: a review. Int J Clin Pract. 2008;62(1):76-87.

23. Zaman Huri H, Fun Wee H. Drug related problems in type 2 diabetes patients with hypertension: a cross-sectional retrospective study. BMC Endocr Disord. 2013;13(1):2.

24. Fog AF, Kvalvaag G, Engedal K, Straand J. Drug-related problems and changes in drug utilization after medication reviews in nursing homes in Oslo, Norway. Scand J Prim Health Care. 2017;35(4):329-35.

25. Al-Taani GM, Al-Azzam SI, Alzoubi KH, Darwish Elhajji FW, Scott MG, Alfahel $\mathrm{H}$, et al. Prediction of drug-related problems in diabetic outpatients in a number of hospitals, using a modeling approach. Drug Healthc Patient Saf. 2017;9:65-70.

26. Singh A, Dwivedi S. Study of adverse drug reactions in patients with diabetes attending a tertiary care hospital in New Delhi, India. Indian J Med Res. 2017:145(2):247-9.

27. Olowofela AO, Isah AO. A profile of adverse effects of antihypertensive medicines in a tertiary care clinic in Nigeria. Ann Afr Med. 2017;16(3):114-9. 